\title{
High Performance Polyurethane Elastomers Using New Cyclaliphatic Diisocyanate
}

\author{
Satoshi Yamasaki ${ }^{*, *}$, Goro Kuwamura ${ }^{*}$, Hirokazu Morita ${ }^{*}$, Daisuke HaSegawA ${ }^{*}$, Ken KoJIO ${ }^{* *, * * *}$, Atsushi Takahara ${ }^{* *, * * *}$ \\ "Specialty Polyurethane materials Development Group, Synthetic Chemicals Laboratory, \\ R\&D Center, Mitsui Chemicals, Inc., 580-32, Nagaura, Sodegaura-City, Chiba 299-0265, Japan \\ ${ }^{* *}$ Institute of Materials Chemistry and Engineering, Kyusyu University \\ *** International Institute for Carbon-Neutral Energy Research, Kyusyu University, 744 Motooka, Nishi-ku Fukuoka 819-0395, Japan \\ (Received : June 20, 2017)
}

\begin{abstract}
Trans-1,4-bis (isocyanatomethyl) cyclohexane (1,4- $\left.\mathrm{H}_{6} \mathrm{XDI}\right)$ has been newly developed as a novel cycloaliphatic diisocyanate with a high symmetric chemical structure. The PUEs were prepared with 1,4- $\mathrm{H}_{6} \mathrm{XDI}$, polyesterpolyol as a long chain polyol and 1,4-butanediol (BD) as a chain extender. As the representative aromatic and aliphatic diisocyanates, 4,4'-diphenylmethane diisocyanate (MDI) and dicyclohexyl methane-4,4'-diisocyanate $\left(\mathrm{H}_{12} \mathrm{MDI}\right)$ were also used as the control. As a result, it was revealed that $1,4-\mathrm{H}_{6}$ XDI-based PUEs achieved high elasticity such as higher impact resilience, lower compression set, improved heat resistant, and good light stability which was not able to be realized with the existing diisocyanates. The reason is explained that the hard segment chains of the 1,4- $\mathrm{H}_{6} \mathrm{XDI}$-based PUEs crystallized well with

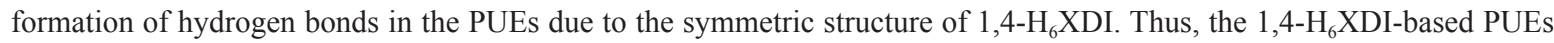
can provide superior properties such as mechanical and thermal properties and excellent light stability compared to existing diisocyanate-based PUEs by rheological evaluation.
\end{abstract}

Key Words: 1,4-Bis (isocyanatomethyl) cyclohexane / Micro-phase separated structure / Polyurethane elastomer / Rheological property

\section{INTRODUCTION}

Most of polyurethane elastomers (PUEs) have been generally produced from three raw materials such as long chain polyol, diisocyanate and short chain polyols (chain extender). Many combinations of these raw materials can control various properties of the PUEs which are composed of alternating hard and soft segments. These segments are microphase-separated into two phases depending on their thermodynamical immiscibility. Thus, the chemical structure of diisocyanate is one of the most important factors to control microphase-separated structure. Diisocyanate can be classified into aromatic, aliphatic, cycloalipatic and aralkyl ones.

The major diisocyanates in the polyurethane industry are aromatic diisocyanates such as $4,4^{\prime}$-diphenyl methane diisocyanate (MDI) and toluene diisocyanate (TDI). MDI is applied to various polyurethane products, in particular, elastomer which requires high resilience, tensile strength and high softening temperature as an indication of heat resistance $^{1,2)}$.

$\dagger$ Corresponding author. Tel: 0438-64-2427, Fax: 0438-64-2349

E-mail : Satoshi.Yamasaki@mitsuichemicals.com
On the other hand, for applications which requires light and weather stabilities, the aromatic diisocyanates mentioned above will not be desirable because polyurethanes prepared by these aromatic diisocyanates become yellowing due to the formation of a quinoid structure of aromatic ring ${ }^{3)}$ if exposed to light for a long time.

For the light stable applications, aliphatic diisocyanates, such as dicyclohexyl methane $-4,4^{\prime}$ - diisocyanate $\left(\mathrm{H}_{12} \mathrm{MDI}\right)^{4)}$, isophorone diisocyanate (IPDI) ${ }^{5}$, hexamethylene diisocyanate $(\mathrm{HDI})^{6)}$, 2,5(2,6)-bis(isocyanatomethyl)bicyclo[2.2.1] heptane $\left(\mathrm{NBDI}^{\mathrm{TM}}\right)^{6}$ ) and 1,3-bis (isocyanatemethyle) cyclohexane $\left(1,3-\mathrm{H}_{6} \mathrm{XDI}\right)^{7}$, have been applied as the raw materials. However, polyurethanes based on these aliphatic diisocyanates exhibits inferior elasticity such as resilience, compression set, strength and dynamic performance and heat resistance $^{1)}$ due to poor cohesive force of resulting hard segment chain.

So the new aliphatic diisocyanate which produces good elastic property, heat resistance and light stability has been demanded from not only industrial but academic points of view. We have developed and commercialized a new cycloaliphatic diisocyanate, trans-1,4-bis (isocyanatomethyl) cyclohexane $\left(1,4-\mathrm{H}_{6} \mathrm{XDI}\right)$ which is referred to as $1,4-\mathrm{H}_{6} \mathrm{XDI}^{8)}$. It is 
expected that $1,4-\mathrm{H}_{6} \mathrm{XDI}$ is applicable to various polyurethane products such as thermoplastic polyurethane elastomer

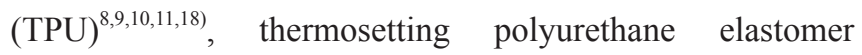
$(\mathrm{TSU})^{8,9,12-14)}$, polyurethane solution ${ }^{8,15)}$, polyisocyanate hardener $^{16)}$, polyurethane dispersion (PUD) ${ }^{15,17)}$ and flexible polyurethane foam $^{8,18)}$.

This paper will report superior properties of polyurethane elastomers based on 1,4- $\mathrm{H}_{6} \mathrm{XDI}$, in particular, mechanical and thermal properties using rheological technique. MDI and $\mathrm{H}_{12} \mathrm{MDI}$-based polyurethane elastomers were employed for comparison. We synthesized two polyurethanes, which are "basic PUE" and "practical PUE". Basic PUE is prepared with a condition of making a TSU. On the contrary, practical PUE is prepared with a condition of making a TPU.

\section{EXPRIMENTAL}

\subsection{Materials}

\section{Long chain polyol}

Polyesterpolyol which is made from the mixture of ethylene glycol and 1,4-butanediol and adipic acid (trade name, U-2024, referred to PEBA-2000, Mitsui Chemicals Inc.) were employed. The number-average molecular weight $\left(M_{\mathrm{n}}\right)$ and hydroxyl number were ca. 2,000 and $56.2 \mathrm{mgKOH} / \mathrm{g}$, respectively.

\section{Diisocyanate}

$1,4-\mathrm{H}_{6} \mathrm{XDI}$ is produced by phosgenation reaction of trans-1,4-bis (diaminomethyl) cyclohexane $\left(1,4-\mathrm{H}_{6} \mathrm{XDA}\right)^{19)}$, which are obtained via nuclear hydrogenation and isomerization reaction of $p$-xylylene diamine by using some catalysts and additives. Figure 1 shows the process for $1,4-\mathrm{H}_{6} X D I$. It has a very linear and symmetrical structure with respect to the isocyanate groups, since it is enriched in the trans isomer. The product is a clear liquid at room temperature with 43.2 of concentration of isocyanate groups ( $\mathrm{NCO} \%)$. The melting point and flash point are ca. 8 and $167^{\circ} \mathrm{C}$, respectively. It can be stored at room temperature.
MDI (4,4'-MDI, MDI-PH, Mitsui Chemicals Inc.) and $\mathrm{H}_{12} \mathrm{MDI}$ (Evonik) were used as the aromatic and aliphatic diisocyanates.

\section{Chain Extender}

1,4-butanediol (1,4-BDO, Aldrich) was used as a chain extender. 1,4-BDO was distillated and dried by bubbling dry nitrogen under a reduced pressure at $100{ }^{\circ} \mathrm{C}$ for $4 \mathrm{~h}$.

\subsection{Preparation for basic and practical PUE}

Basic PUEs were made by a prepolymer method. Basic PUE in this article is also called thermoset polyurethane elastomer. The isocyanate-terminated urethane prepolymers were prepared from a wide variety of combination of long chain polyol and diisocyanate with various equivalent of [ $\mathrm{NCO}] /$ $[\mathrm{OH}]\left(\mathrm{NCO}\right.$ index) at ca. $80{ }^{\circ} \mathrm{C}$ until achievement of target $\mathrm{NCO} \%$ under a nitrogen atmosphere. The reaction was pursued by an amine equivalent method. The prepolymer and 1,4-BOD were set to be $80^{\circ} \mathrm{C}$ and then mixed well for ca. $10 \mathrm{~min}$ at $80^{\circ} \mathrm{C}$ at stoichiometry of 1.02 at $[\mathrm{NCO}]_{\text {prepolymer }} /$ $[\mathrm{OH}]_{1,4-\mathrm{BDO}}$ after addition of catalyst (Dibutyltin dilaurate, DBTDL, Aldrich) and anti-oxidant bis[3-(3-tert-butyl-4-hydroxy-5-methylphenyl) propionic acid], BASF). The reaction mixture was poured into a stainless steel mold with $2 \mathrm{~mm}$ of thickness. The curing condition was at $100{ }^{\circ} \mathrm{C}$ for 22 hours and then annealed at $23{ }^{\circ} \mathrm{C}$ and $55 \%$ relative humidity for 7 days. PUEs with various different hardness were prepared by changing the concentration of the hard segment composed of the diisocyanate and 1,4-BDO.

On the contrary, practical PUEs were also prepared by prepolymer method at a batch procedure for the purpose of investigating the potential of $1,4-\mathrm{H}_{6}$ XDI in TPU application. Procedure of practical PUE was as follows. The isocyanate-terminated urethane prepolymers were also synthesized the same procedure at preparation of PUEs. After the temperatures of prepolymer and 1,4-BDO were set to be $80^{\circ} \mathrm{C}$, the prepolymer and 1,4-BDO were mixed well with the ratios of $[\mathrm{NCO}]_{\text {prepolymer }} /[\mathrm{OH}]_{1,4-\mathrm{BD}}=1.01$ at $80{ }^{\circ} \mathrm{C}$ for $10 \mathrm{~min}$ after

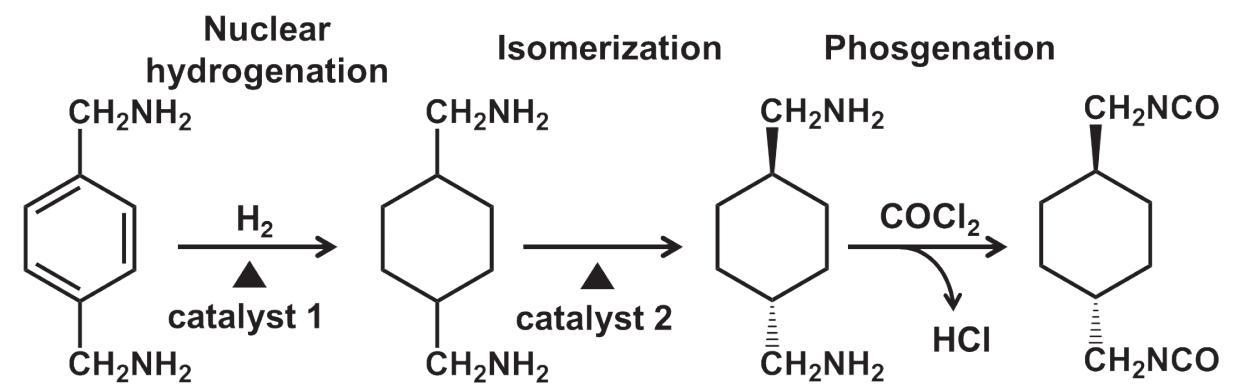

Fig. 1. The production process for $1,4-\mathrm{H}_{6} \mathrm{XDI}$. 
addition of catalyst (DBTDL), anti-oxidant bis[3-(3-tert-butyl-4-hydroxy-5-methylphenyl) propionic acid], BASF) and hindered amine light stabilizer (bis(1,2,2,6,6-pentamethyl-4-piperidyl) sebacate, ADEKA) under a nitrogen atmosphere. Then the reaction mixture was poured into the Teflon ${ }^{\circledR}$ coating container and then cured for 2 hours at $130^{\circ} \mathrm{C}$ and 20 hours at $110^{\circ} \mathrm{C}$ to obtain a polyurethane sheet. Next, the polyurethane sheet was cut and granulated. After drying the TPU (practical PUE) granule, TPU pellets were prepared by a uniaxial extrusion at the range from $200{ }^{\circ} \mathrm{C}$ to $235^{\circ} \mathrm{C}$ of cylinder temperature.

The TPU pellets obtained were dried in an oven at $80{ }^{\circ} \mathrm{C}$ for $12 \mathrm{~h}$ at a reduced pressure. The TPU sheets were made by injection molding operated by NEX-140 (Injection machine, $\mathrm{D}=40 \mathrm{~mm}, \mathrm{~L} / \mathrm{D}=24$, NISSEI PLASTIC INDUSTRIAL) with the cylinder temperature range from $200{ }^{\circ} \mathrm{C}$ to $220{ }^{\circ} \mathrm{C}$ at injection speed of $60 \mathrm{~mm} \mathrm{~s}^{-1}$ and back pressure range from $10 \mathrm{MPa}$ to $20 \mathrm{MPa}$. TPU sheets were stored at $23{ }^{\circ} \mathrm{C}$ and $55 \%$ relative humidity for at least one week.

\subsection{Characterization}

\section{Mechanical and physical properties}

Hardness, tensile test, tear strength and impact resilience, were measured by JIS K-7311 and JIS K-7312, respectively.

In particular, tensile test of the basic PUE and injection molding sheet prepared by TPU pellet was performed with an Instron type tensile tester (model U4410/U4400, Toyo Seiki Co., LTD.) equipped a device monitoring sample length at $23{ }^{\circ} \mathrm{C}$ and $55 \%$ relative humidity. The samples were prepared with a steel stamping die (dumbbell type JIS No. 3). An initial length and elongation velocity were set to $20 \mathrm{~mm}$ and $300 \mathrm{~mm} \mathrm{~min}^{-1}$ according to JIS K-7311 and $500 \mathrm{~mm} \mathrm{~min}^{-1}$ according to JIS K-7312, respectively.

\section{Thermal Mechanical Analysis (TMA)}

The softening temperature of PUE and injection molding sheet prepared by TPU pellet was measured by using TMA (model TMA/SS6100, Seiko Instruments) according to JIS K-7196. The softening temperature was determined at temperatures ranging from -60 to $260{ }^{\circ} \mathrm{C}$ with a heating rate of $5{ }^{\circ} \mathrm{C} \mathrm{min}^{-1}$ and load of $500 \mathrm{mN}$ under a nitrogen atmosphere.

\section{Differential scanning calorimetry (DSC)}

The thermal behavior was determined using DSC (model DSC6200, Seiko Instruments). DSC thermograms were obtained at temperatures ranging from -100 to $250{ }^{\circ} \mathrm{C}$ with a heating rate of $10{ }^{\circ} \mathrm{C} \mathrm{min}^{-1}$ under a nitrogen atmosphere. Each sample was cooled at $-100{ }^{\circ} \mathrm{C}$ and after being held at $-100{ }^{\circ} \mathrm{C}$ for $10 \mathrm{~min}$, measurements were carried out up to $270{ }^{\circ} \mathrm{C}$. The sample weighed $8-10 \mathrm{mg}$ and indium was used to calibrate the DSC cell.

\section{Dynamic viscoelastic analysis (DMA)}

The temperature dependence of the dynamic viscoelastic properties of injection molding sheet prepared by TPU pellet was measured using a Rheometrics mechanical spectrometer (model RSA III, TA instruments) at temperatures ranging from -80 to $200{ }^{\circ} \mathrm{C}$, at a heating rate of $3{ }^{\circ} \mathrm{C} \mathrm{min}{ }^{-1}$ and a frequency of $1 \mathrm{~Hz}$ under a nitrogen atmosphere.

\section{Wide angle X-ray scattering (WAXD) and Small angle X-ray scattering (SAXS)}

The WAXD profile of PUE was measured by a Rigaku RINT2550. The WAXD intensity distribution was measured with a rotating-anode X-ray generator (model Rigaku Ultrax-18, operated at $40 \mathrm{kV}$ and $370 \mathrm{~mA}$ ). The intensity was measured by a scintillation counter. Ni-filtered $\mathrm{Cu} \mathrm{K} \alpha$ radiation was used as an incident $\mathrm{X}$-ray source. The Gonio radius and scan speed were set to be $185 \mathrm{~mm}$ and $2^{\circ} \mathrm{min}^{-1}$, respectively.

The SAXS profile of PUE was measured by a Rigaku RINT2500 with a three-slit system. The SAXS intensity distribution was measured with a rotating-anode X-ray generator (model Rigaku Ultrax-18, operated at $50 \mathrm{kV}$ and $300 \mathrm{~mA}$ ). The intensity was measured by a position sensitive proportional counter. The X-ray source was monochromatized to $\mathrm{Cu}$ $\mathrm{K} \alpha$ radiation. The camera length and exposure time were set to be $990 \mathrm{~mm}$ and $3600 \mathrm{sec}$, respectively.

\section{Light stability}

The light stability of TPU films with ca. $100 \mu \mathrm{m}$ thickness were evaluated as $\Delta \mathrm{E}$ using a xenon weather meter (Model: Super xenon weather meter SX-75, Suga Test Instruments) with xenon exposure irradiation of $100 \mathrm{~W} / \mathrm{m}^{2}$ at black panel temperature of $89{ }^{\circ} \mathrm{C}$ and $50 \%$ relative humidity. $\Delta \mathrm{E}$ is the color difference of each film before and after xenon exposure test. It was measured by a spectral color difference meter (Model: SD7000, Nippon Denshoku Industry, Co., Ltd.).

\section{Demold time at injection molding}

The demold time of TPU sheets was evaluated using the injection molding machine described at the above section. The demold time was defined as the time that the mold 
shrinkage rate of injection molded sheet was set within $2 \%$. The rate was calculated by monitoring each vertical and horizontal length of the sheet right after injection molding.

\section{RESULTS AND DISCUSSION}

\subsection{Rheological properties of polyurethane based on $1,4-\mathrm{H}_{6} \mathrm{XDI}$}

The rheological properties of basic PUEs prepared with three different diisocyanate, MDI as the aromatic diisocyanate, $\mathrm{H}_{12} \mathrm{MDI}$ as the aliphatic diisocyanate and $1,4-\mathrm{H}_{6} \mathrm{XDI}$ with a PEBA-2000 and 1,4-BDO were investigated. Figure 2 shows impact resilience versus hardness of three different diisocyanate-based PUEs.

As one can see from this data, impact resilience of 1,4- $\mathrm{H}_{6}$ XDI- based polyurethanes were higher than those of MDI and $\mathrm{H}_{12}$ MDI based ones over all range of hardness. 1,4- $\mathrm{H}_{6}$ XDI- based polyurethanes showed excellent impact resilience of ca. $60 \%$ even at $95 \mathrm{~A}$ of hardness.

Figure 3 shows softening temperature as an index of heat resistance versus $100 \%$ modulus of three different diisocyanate based PUEs. The hard segment concentration (HSC) of all PUEs was $15 \%$. HSC was calculated the following equation (1).

$$
\mathrm{HSC}(\%)=\left[\frac{C E(g)+N C O(g) \times \frac{C E(\text { eq. })}{P o l y o l(e q .)+C E(e q .)}}{N C O(g)+P o l y o l(g)+C E(g)}\right] \times 100
$$

, where $C E(g)$ is the weight of chain extender, $N C O(g)$ is the weight of diisocyanate, Polyol $(g)$ is the weight of long chain polyol, $C E($ eq.) is the equivalent weight of chain extender, $\mathrm{NCO}($ eq.) is the equivalent weight of diisocyanate, Polyol(eq.)

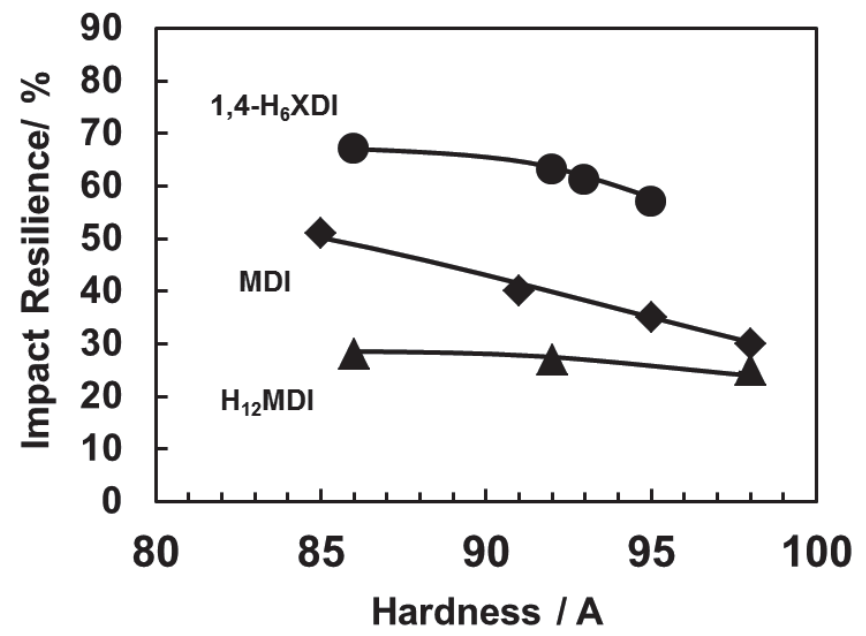

Fig. 2. Impact resilience versus hardness of three different diisocyanatebased PUEs. is the equivalent weight of long chain polyol $1,4-\mathrm{H}_{6} \mathrm{XDI}-$ based polyurethane showed higher softening temperature and $100 \%$ modulus simultaneously compared to those of MDI and $\mathrm{H}_{12} \mathrm{MDI}$ - based ones at the same HSC of $15 \%$. With the same HSC, if the $100 \%$ modulus and softening temperature of polyurethane are high, the elasticity and heat resistance are considered to be excellent. From Fig. 3, 1,4-H XDI- based polyurethane showed superior elasticity and heat resistance compared to MDI and $\mathrm{H}_{12} \mathrm{MDI}$ - based ones.

Figure 4 shows light stability of thin polyurethane films based on MDI and 1,4- $\mathrm{H}_{6}$ XDI with $100 \mu \mathrm{m}$ of thickness by xenon exposure irradiation of $100 \mathrm{~W} / \mathrm{m}^{2}$ at black panel temperature of $89{ }^{\circ} \mathrm{C}$ and $50 \%$ relative humidity. $\Delta \mathrm{E}$ of $\mathrm{H}_{12} \mathrm{MDI}-$ based polyurethane film could not be plotted in this figure, since this film was molten during this test because of the low softening temperature of $\mathrm{H}_{12} \mathrm{MDI}$ - based polyurethane. From xenon exposure test, $\Delta \mathrm{E}$ of $1,4-\mathrm{H}_{6} \mathrm{XDI}$ - based polyurethane was below 0.3 over 10 days, so it showed excellent light stability.

1,4- $\mathrm{H}_{6}$ XDI- based polyurethane showed excellent elasticity, heat resistance and light stability compared to those of MDI and $\mathrm{H}_{12} \mathrm{MDI}$, as expected from the molecular structure of this diisocyanate. In order to classify the difference in the superstructure of various diisocyanate-based polyurethanes, the measurement of DSC for PUEs were carried out. The glass transition temperature $\left(T_{\mathrm{g}, \mathrm{s}}\right)$ of soft segment phase of $1,4-\mathrm{H}_{6} \mathrm{XDI}-$ based polyurethane was observed at $-55.4{ }^{\circ} \mathrm{C}$ and then those of MDI and $\mathrm{H}_{12} \mathrm{MDI}$ were observed at $-53.5^{\circ} \mathrm{C}$ and $-52.2{ }^{\circ} \mathrm{C}$, respectively. On the contrary, the endothermic temperature observed at relatively high temperature region was defined as the melting point of crystallized hard segment phase. The melting point $\left(T_{\mathrm{m}, \mathrm{h}}\right)$ of the hard segment phase of

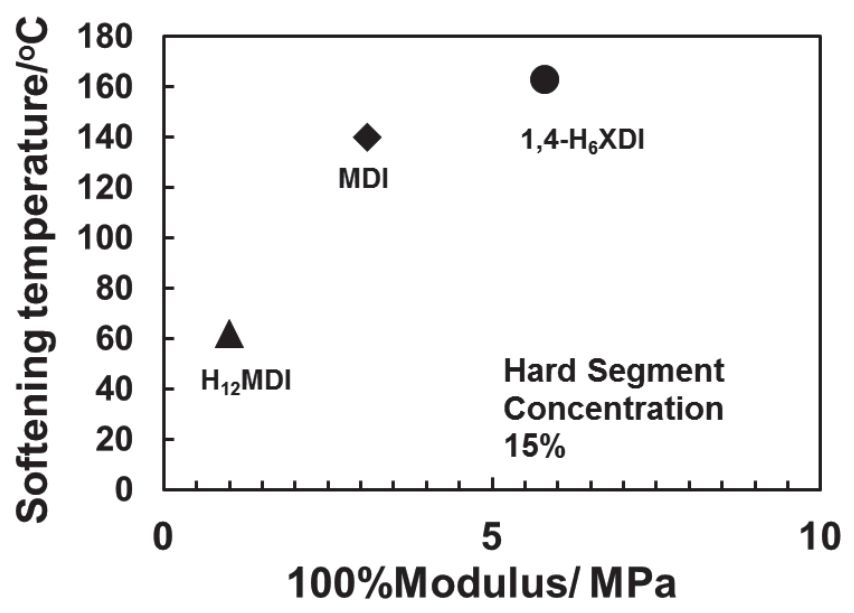

Fig. 3. Softening temperature versus $100 \%$ modulus of three different diisocyanate- based PUEs with hard segment concentration of $15 \mathrm{wt} \%$. 
1,4- $\mathrm{H}_{6}$ XDI- based polyurethane was observed at $170.2^{\circ} \mathrm{C}$ and then those of MDI and $\mathrm{H}_{12} \mathrm{MDI}$ were observed at $152.5^{\circ} \mathrm{C}$ and $67.6^{\circ} \mathrm{C}$, respectively. The degree of microphase-separated structure of these polyurethanes must be associated with the difference in $T_{\mathrm{g}, \mathrm{s}}$ and $T_{\mathrm{m}, \mathrm{h}},\left|T_{\mathrm{g}, \mathrm{s}}-T_{\mathrm{m}, \mathrm{h}}\right|$. In other words, the degree of microphase-separated structure might be progressed with increasing the value of $\left|T_{\mathrm{g}, \mathrm{s}}-T_{\mathrm{m}, \mathrm{h}}\right|$.

Figure 5 shows a comparison with the degree of microphase separation of various diisocyanate- based polyurethanes. The $\left|T_{\mathrm{g}, \mathrm{s}}-T_{\mathrm{m}, \mathrm{h}}\right|$ of $1,4-\mathrm{H}_{6}$ XDI- based PUE was the highest value of $226^{\circ} \mathrm{C}$ and those of MDI and $\mathrm{H}_{12} \mathrm{MDI}$ were $206^{\circ} \mathrm{C}$ and $119.8^{\circ} \mathrm{C}$, respectively.

Figure 6 (a) and (b) show the WAXD and SAXS profiles for three different diisocyanate- based polyurethanes, respectively. 1,4- $\mathrm{H}_{6}$ XDI- based PUE only exhibited crystalline peaks at $2 \Theta=17,19,20$ and 24 degree which can be assigned to the crystallized hard segment domains in the WAXD profiles (Fig. 6 (a)) ${ }^{21}$. The observation at these crystalline peaks from the hard segment domains are quite rare ${ }^{22)}$. These results imply that hard segment chains form rich phases and packed well each other in the PUE.

On the contrary, obvious peaks was observed for 1,4- $\mathrm{H}_{6}$ XDI- based PUE in SAXS profile. This peak can be assigned to interdomain spacing of hard segment domains in the microphase-separated structure ${ }^{21,23)}$. This indicates that well-developed microphase-separated structure was formed for $1,4-\mathrm{H}_{6}$ XDI- based PUE. SAXS profiles for MDI-based PUE also exhibited strong intensity at low scattering angle region. This result implies that MDI-based PUE also possess developed microphase separated structure. The peak for 1,4-H $\mathrm{H}_{6}$ XDI- based PUE was observed slightly higher scattering angle region, interdomain spacing was smaller than for MDI-based polyurethane. For $\mathrm{H}_{12} \mathrm{MDI}$-based PUE, since there are no clear scattering peak in the SAXS profile, indicating the phase mixing trend of this PUE.

From DSC measurement and X-ray scattering patterns as shown in Figs. 5 and 6, the micro phase-separated structure between soft segment and hard segment phases of 1,4- $\mathrm{H}_{6} \mathrm{XDI}$ - based PUE might be progressed compared to those of MDI and $\mathrm{H}_{12} \mathrm{MDI}$, respectively.

Figure 7 shows the schematic model of super structure of 1,4- $\mathrm{H}_{6}$ XDI- based polyurethane. Since 1,4- $\mathrm{H}_{6}$ XDI has a high symmetrical structure, resulting hard segment chains form a well-organized hard segment phase by reaction with a chain extender, such as 1,4-BDO. Therefore, a micro-phase separated structure between soft segment and hard segment phases is progressed, resulting in a superior elasticity and heat resistance such as softening temperature.

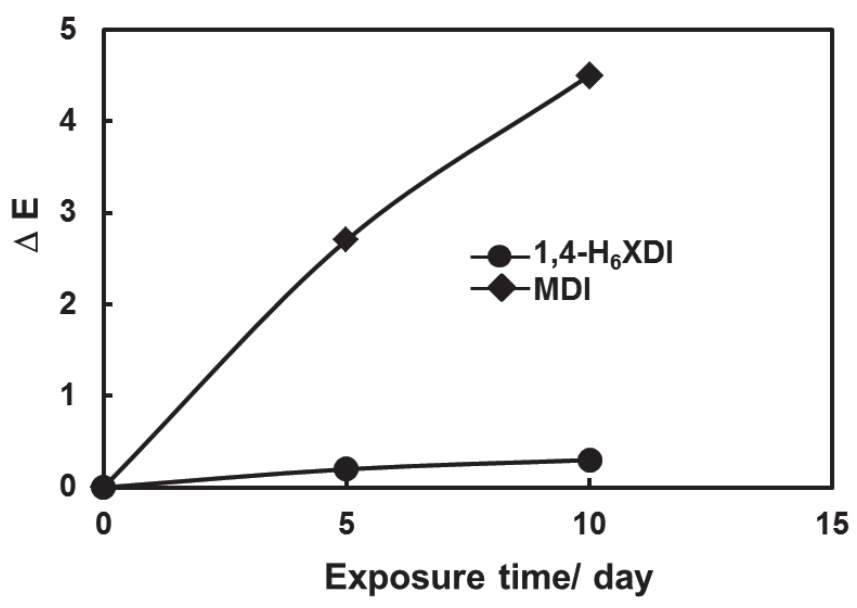

Fig. 4. Time dependence of $\Delta \mathrm{E}$ of MDI- based and $1,4-\mathrm{H}_{6} \mathrm{XDI}-$ based TPU films by xenon exposure.

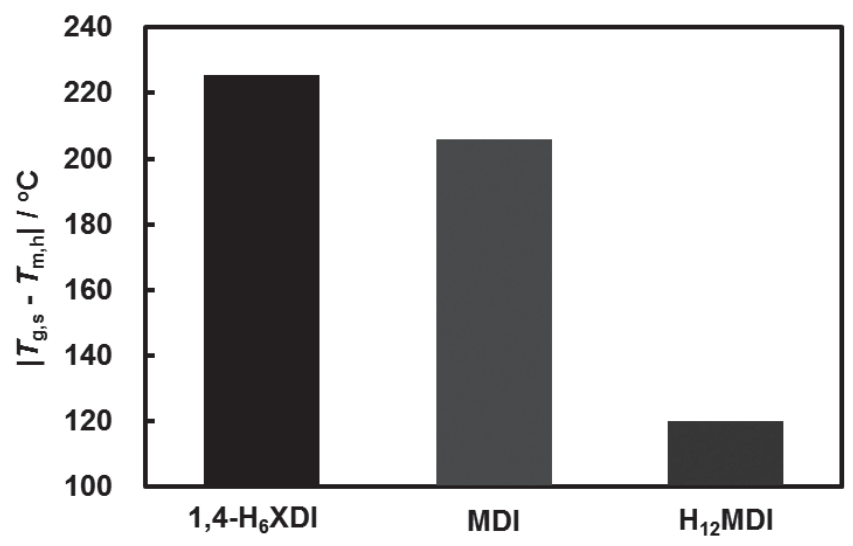

Fig. 5. Comparison with the degree of micro- phase separation of various diisocyanate-based PUEs.
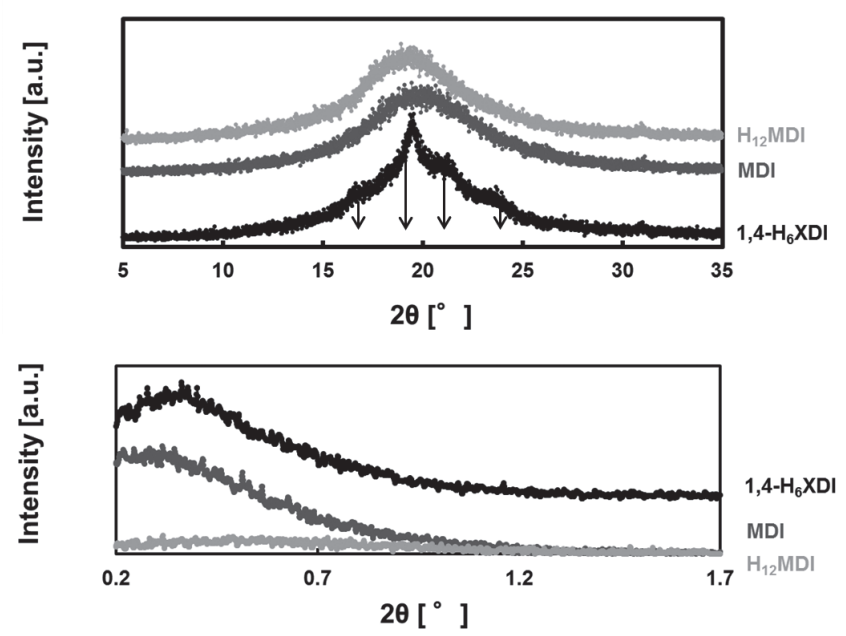

Fig. 6. (a) WAXD profiles (b) SAXS profiles for three different diisocyanate-based PUEs. 


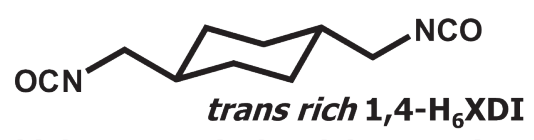

high symmetrical cyclohexane ring

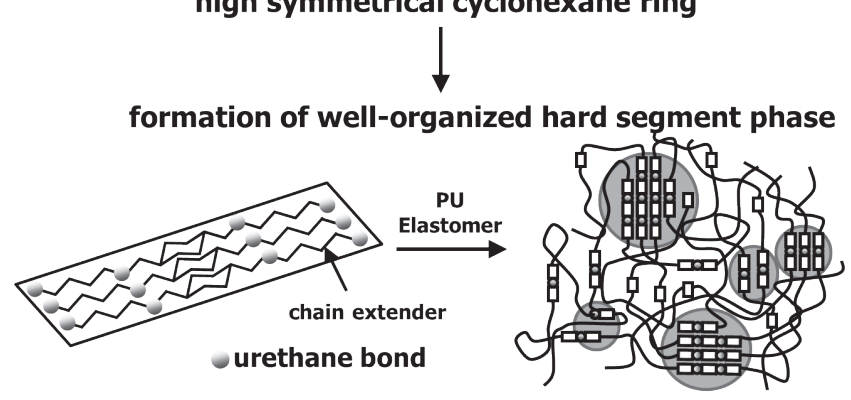

Fig. 7. Schematic model of super structure of $1,4-\mathrm{H}_{6}$ XDI-based PUE.

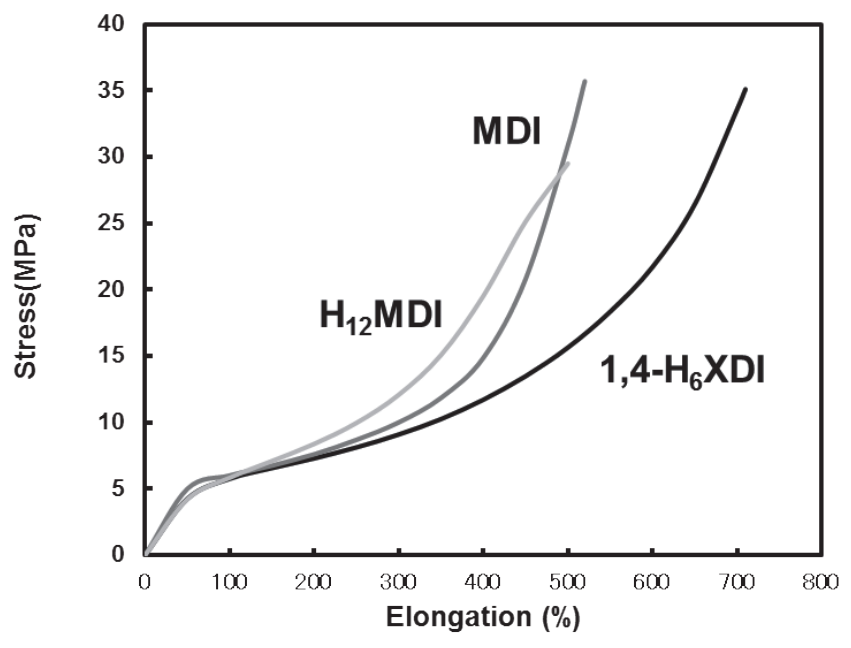

Fig. 8. Stress-strain curves for three different diisocyanate-based TPUs.

next was MDI-TPU, finally 1,4-H $\mathrm{H}_{6} \mathrm{XI}-\mathrm{TPU}$, respectively. In other words, 1,4- $\mathrm{H}_{6} \mathrm{XDI}-\mathrm{TPU}$ showed excellent heat resistance compared to other TPUs. These trends may be associated with the strong microphase separation of $1,4-\mathrm{H}_{6} \mathrm{XDI}-$ based TPU.

In the soft segment glass transition region, 1,4- $\mathrm{H}_{6} \mathrm{XDI}-$ based TPU had a significant shoulder which is likely recrystallization and melting of crystallized PEBA of soft segment.

Next, the features of $1,4-\mathrm{H}_{6}$ XDI- based TPU are described from the industrial point of view.

Figure 10 shows the formulation and comparison among the compositions of TPUs using various diisocyanates with same hardness of $85 \mathrm{~A}$. Since $1,4-\mathrm{H}_{6}$ XDI- based TPU showed high elasticity even at small amount of diisocyanate than those of $\mathrm{H}_{12} \mathrm{MDI}$ and MDI, the consumption of this diisocyanate could be reduced by half ( 0.5 times $)$ of $\mathrm{H}_{12} \mathrm{MDI}$ system and 0.6 times of MDI system, when preparing TPUs with the same hardness.

Figure 11 shows the comparison of demold time at injection molding of various TPUs. The processability of TPU article prepared by injection molding is judged to be good when the demold time was shorter. 1,4- $\mathrm{H}_{6}$ XDI- based TPU showed shortest demold time due to the faster solidification from molten state, since the hard segment phase composed from 1,4- $\mathrm{H}_{6} \mathrm{XDI}$ aggregated faster than those of $\mathrm{H}_{12} \mathrm{MDI}$ and MDI even at cooling process evaluated by DSC measurement. The elasticity of $1,4-\mathrm{H}_{6} \mathrm{XDI}$ - based TPU achieved to settle quickly compared with other TPUs because of faster aggregation of the hard segment phase.

After injection molding, the hardness of TPU sheets were measured every other day at $23{ }^{\circ} \mathrm{C}$ and $55 \%$ relative humidity. Figure 12 shows the aging time dependence of hardness after injection molding. 1,4- $\mathrm{H}_{6} \mathrm{XDI}$ - based TPU 
Table I. Mechanical Properties of 1,4- $\mathrm{H}_{6}$ XDI and other isocyanate based TPUs

\begin{tabular}{|c|c|c|c|c|}
\hline Properties & unit & 1,4-H XDI-TPU & $\mathrm{H}_{12} \mathrm{MDI}-\mathrm{TPU}$ & MDI-TPU \\
\hline Hardness & A & 86 & 85 & 87 \\
\hline Impact resilience & $\%$ & 69 & 30 & 51 \\
\hline $\begin{array}{l}\text { Compression set } \\
23^{\circ} \mathrm{C} / 70^{\circ} \mathrm{C}\end{array}$ & $\% / \%$ & $14.7 / 30.0$ & $17.3 / 73.5$ & $16.7 / 38.7$ \\
\hline$T_{\mathrm{g}}$ & ${ }^{\circ} \mathrm{C}$ & -46 & 23 & -27 \\
\hline $\begin{array}{l}\text { Softening } \\
\text { temperature (TMA) }\end{array}$ & ${ }^{\circ} \mathrm{C}$ & 171 & 105.6 & 148.4 \\
\hline $100 \%$ modulus & $\mathrm{MPa}$ & 5.8 & 5.8 & 6 \\
\hline $300 \%$ modulus & $\mathrm{MPa}$ & 9.1 & 12.1 & 10 \\
\hline Tensile strength & $\mathrm{MPa}$ & 35.1 & 29.5 & 35.7 \\
\hline Elongation at break & $\%$ & 710 & 500 & 520 \\
\hline Tear strength & $\mathrm{kN} / \mathrm{m}$ & 118 & 101 & 102 \\
\hline
\end{tabular}

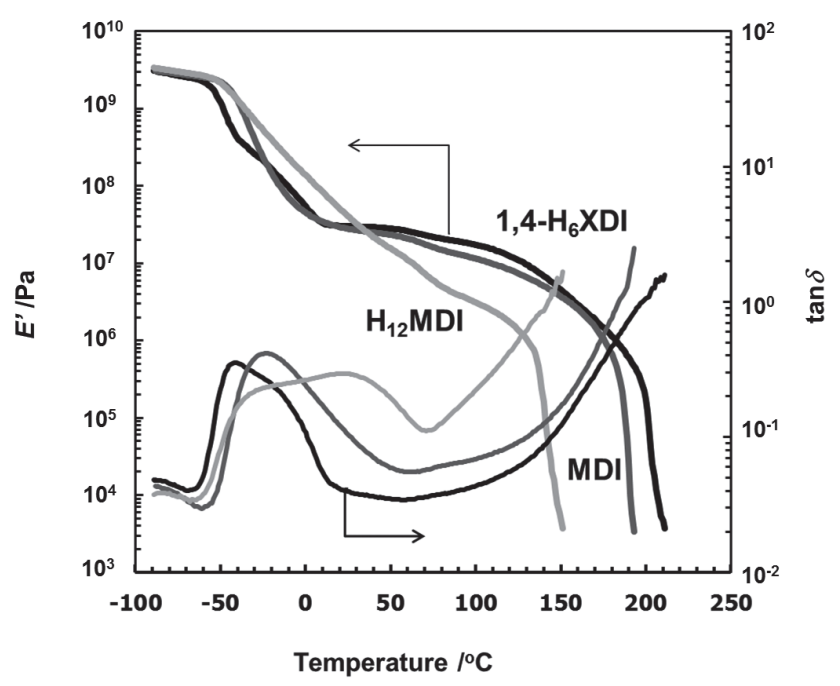

Fig. 9. Temperature dependence of dynamic storage modulus $\left(E^{\prime}\right)$ and $\tan \delta$ for three different diisocyanate-based TPUs.

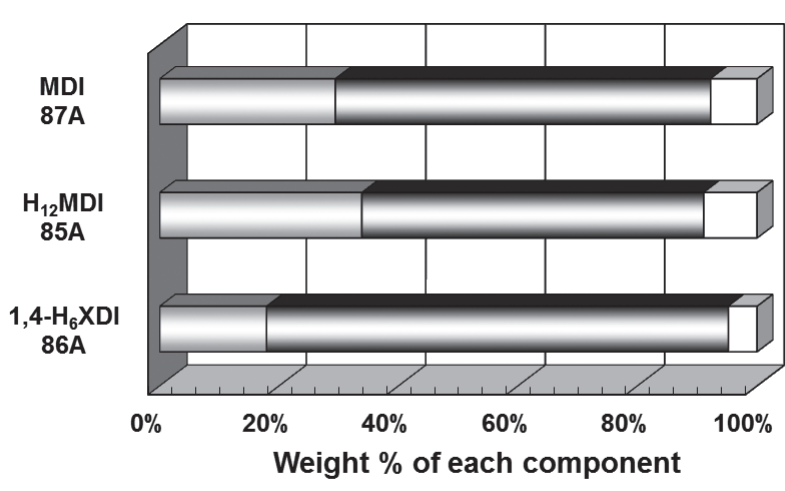

口diisocyanate $\square$ Polyesterpolyol\#2000 $\square$ chain extender

Fig. 10. Formulation and comparison among the compositions of TPUs using various diisocyanates with same hardness of $85 \mathrm{~A}$.

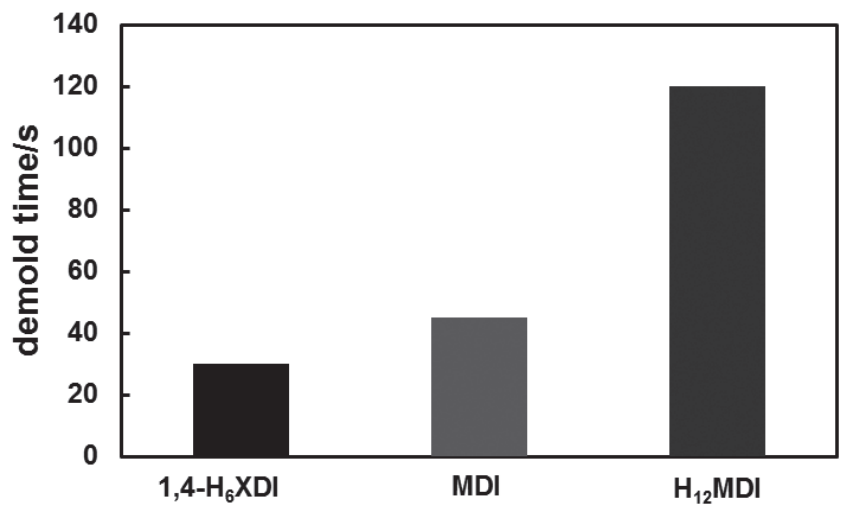

Fig. 11. Comparison of demold time at injection molding of various TPUs.

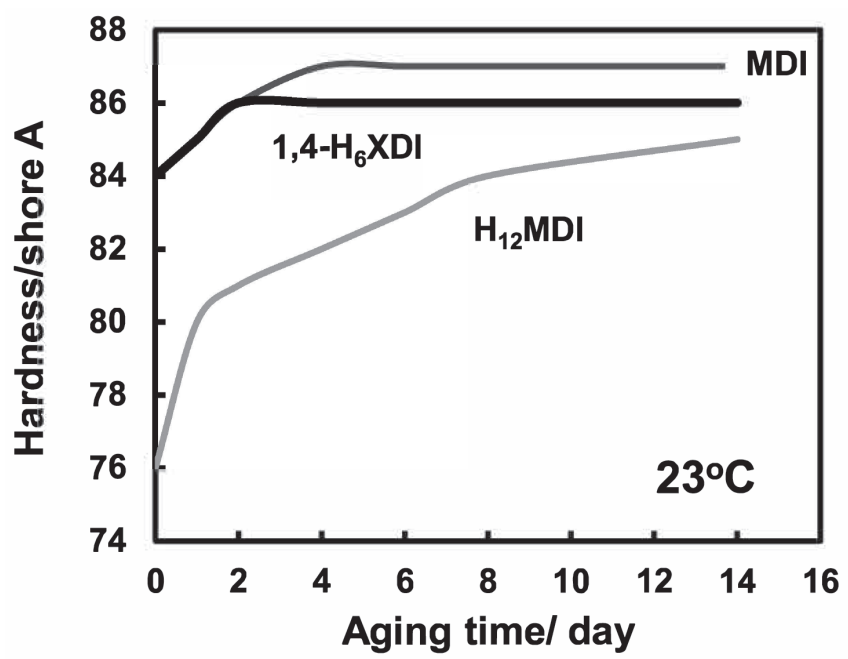

Fig. 12. Aging time dependence of hardness for three different diisocyanate-based TPUs. 
achieved constant hardness quickly compared to MDI and $\mathrm{H}_{12} \mathrm{MDI}$-based TPUs. This result was contributed to the same mechanism as mentioned above.

In summary, 1,4- $\mathrm{H}_{6}$ XDI- based TPU shows non-yellowing, high elasticity, such as low $\tan \delta$, low compression set and high impact resilience. They have high heat resilience such as a high softening temperature and high productivity such as short demold time and fast achievement of designed hardness compared to the conventional diisocyanate- based TPUs.

\section{CONCLUSIONS}

New cycloaliphatic diisocyanate, 1,4- $\mathrm{H}_{6} \mathrm{XDI}$, gives high-performance to polyurethane elastomers. This new aliphatic diisocyanate with a high symmetrical structure results in a superior aggregation of hard segment reacted with 1,4BDO, giving a high softening temperature and forms well organized micro-separated structure from the experimental results of WAXD, SAXS, DSC and DMA. So that polyurethane based on 1,4- $\mathrm{H}_{6} \mathrm{XDI}$ achieve high elasticity, heat resistant and light stability which was not able to be realized with the conventional diisocyanate. For TPU application, the mechanical and thermal properties of $1,4-\mathrm{H}_{6} \mathrm{XDI}$ - based TPU are superior to those based on MDI and $\mathrm{H}_{12} \mathrm{MDI}$, in particular, low $\tan \delta$, low glass transition temperature, low compression set, high softening temperature, high impact resilience and high tear strength. In addition, 1,4- $\mathrm{H}_{6} \mathrm{XDI}-$ based TPU gives high productivity such as short demold time and fast achievement of designed hardness.

These results support that elastomer systems based on 1,4- $\mathrm{H}_{6}$ XDI shows superior mechanical and thermal properties and excellent light stability, dynamic fatigue performance as a high performance PUEs.

\section{ACKNOWLEGMENTS}

The authors acknowledge Dr. Kazuki Mita of Mitsui Chemicals Inc. and Mr. Yasuhiro Hanamoto of Mitsui Chemicals Analysis Center Inc. for measuring and discussing the data using WAXD and SAXS.

\section{REFERENCES}

1) Oertel G, "Polyurethane Handbook" (1994) Hanser Press, Munich.
2) Ulrich H, "Chemistry and Technology of Isocyanate" (1996) John Wiley \& Sons Inc, England.

3) Lemaire J, Gardette J L, Rivaton A, Roger A, Polym Degrad Stab, 15, 1, (1986).

4) Hespe H F, Zembrod A, Cama F J, Lantman C W, Seneker S D, J Appl Polym Sci, 44, 2029 (1992).

5) Pandya M V, Deshpande D D, Hundiwale D G, J Appl Polym Sci, 32, 4959 (1986).

6) Kojio K, Nakashima S, Furukawa M, Polymer, 48, 997 (2007).

7) Xie R, Bhattacharjee D, Argyropoulos J, J Appl Polym Sci, 113, 839 (2009).

8) Kuwamura G, Nakagawa T, Hasegawa D, Yamasaki S, US Patent 8,722,752B2 2014-05-13.

9) Petrovic S Z, Ferguson J, Prog Polym Sci, 16, 695 (1991).

10) Woodward J, Merino D, Greenland B, Hamley I, Light Z, Slark A, Wayne H, Macromolecules, 43, 2512 (2010).

11) Yamasaki S, Nishiguchi D, Kojio K, Furukawa M, Polymer, 48, 4793 (2007).

12) Abouzahr S, Wilkes G L, Ch.5 in "Processing, Structure and Properties of Block Copolymers", (1985), Elesevier, New York.

13) Gibson PE, Vallance MA, Cooper S L, Ch.6 in "Developments in Block Copolymers-1", (1982), Appl. Sci. publishers, New York.

14) Stephen $S$, Robert $C$, Jordan $D$, Yamasaki S, Nakagawa $T$, Hasegawa D, Center of Polyurethane Industry Conference (2015).

15) Dieterich D, Prog Org Coat, 9, 281 (1981).

16) Richter F, Schmitz J, Munsdstock H, Pires R, International Waterborne, High-Solids and Coatings Symposium, 71 (2003).

17) Geurink P, Scherer T, Buter R, Steenbergen A, Henderiks H, Prog Org Coat, 55, 119 (2006).

18) Sonoda K, Ishibashi K, Ito H, Yoshii N, Center of Polyurethane Industry Conference (2010).

19) Yoshimura N, Hamada T, Watanabe E, Morooka S, US Patent 8,865,938B1 2014-10-21.

20) Hepburn C, "Polyurethane Elastomers" $2^{\text {nd }} e d .$, (1992), Elesevier Science Publishers, England.

21) Nozaki S, Masuda S, Kamitani K, Kojio K, Takahara A, Kuwamura G, Hasegawa D, Moorthi K, Mita K, Yamasaki S, Macromolecules 50, 1008 (2017).

22) Kojio K, Nakamura S, Furukawa M, J Polym Sci, Part B, Polym Phys 46(19), 2054 (2008).

23) Kojio K, Matsuo K, Motokucho S, Yoshinaga K, Shimodaira Y, Kimura K, Polym J 43, 692 (2011). 